PROCEEDINGS OF THE

AMERICAN MATHEMATICAL SOCIETY

Volume 137, Number 2, February 2009, Pages 553-562

S 0002-9939(08)09627-5

Article electronically published on August 19, 2008

\title{
CHORD THEOREMS ON GRAPHS
}

\author{
MOHAMMAD JAVAHERI
}

(Communicated by Jim Haglund)

\begin{abstract}
The chord set of a function $f: \mathbb{R} \rightarrow \mathbb{R}$, denoted by $H(f)$, is the set of $r \in \mathbb{R}$ such that there exists $x \in \mathbb{R}$ with $f(x+r)=f(x)$. It is known that if $f$ is a continuous periodic function, then it has every chord, i.e. $H(f)=\mathbb{R}$. Equivalently, if $f$ is a real-valued Riemann-integrable function on the unit circle $C$ with $\int_{C} f=0$, then for any $r \in[0,1]$, there exists an $\operatorname{arc} L$ of length $r$ such that $\int_{L} f=0$. In this paper, we formulate a definition of the chord set that gives way to generalizations on graphs. Given a connected finite graph $G$, we say $r \in H(G)$ if for any function $f \in L^{1}(G)$ with $\int_{G} f=0$ there exists a connected subset $A$ of size $r$ such that $\int_{A} f=0$. Among our results, we show that if $G$ has no vertex of degree 1 , then $[0, l(G)] \subseteq H(G)$, where $l(G)$ is the length of the shortest closed path in $G$. Moreover, we show that if every vertex of a connected locally finite graph has even degree, then the graph has every chord.
\end{abstract}

\section{INTRODUCTION}

Let $G=(V, E)$ be a connected graph, possibly with loops or multiple edges, where $V$ denotes the set of vertices and $E$ denotes the set of edges of $G$. We can think of $G$ as a measure space $\left(G, \mu_{G}\right)$ by identifying each edge with the interval $[0,1]$ with the standard uniform measure. Let $L^{1}(G)$ denote the set of real-valued functions on $G$ that are Riemann-integrable when restricted to each edge of $G$.

The identification of each edge of $G$ with the interval $[0,1]$ also turns $G$ into a topological space: a subset of $G$ is closed if its intersection with each edge of $G$ is closed. Let $X(G)$ denote the set of closed and connected subsets of $G$. Given $r \in[0,|E|]$, we let

$$
X_{r}(G)=\left\{u \in X(G): \mu_{G}(u)=r\right\} .
$$

Definition 0.1. The partition set of a connected graph $G$ is the set of real numbers $r$ such that there exists $n \in \mathbb{N}$ and a collection $\left\{U_{\alpha}\right\}_{1}^{k} \subset X_{r}(G)$ such that, except for a finite number of points, every point of $G$ appears in exactly $n$ members of the collection. We denote the partition set of $G$ by $P(G)$.

The chord set of a connected graph $G$ is the set of real numbers $r$ such that for every $f \in L^{1}(G)$ with $\int_{G} f d \mu_{G}=0$ there exists $U \in X_{r}(G)$ such that $\int_{U} f d \mu_{G}=0$. We denote the chord set of $G$ by $H(G)$.

Received by the editors January 22, 2008.

2000 Mathematics Subject Classification. Primary 28A99; Secondary 05C99.

Key words and phrases. Chord theorems, Euler graphs, chord set of locally finite graphs.

(C)2008 American Mathematical Society 
It is worth mentioning how our definition of a chord set relates to the definition of the chord set of a function. Recall that the chord set of a function $F: \mathbb{R} \rightarrow \mathbb{R}$ is defined by

$$
H(F)=\{r \in \mathbb{R} \mid \exists x \in \mathbb{R}: F(x+r)=F(x)\} .
$$

Let $G_{0}$ be the graph with one vertex and one loop. Any $f: G_{0} \rightarrow \mathbb{R}$ induces a periodic function $p$ on $\mathbb{R}$ with period one. If $\int_{G_{0}} f=0$, then $F(x)=\int_{0}^{x} p(t) d t$ is periodic with period one. Since every continuous periodic function has every chord (see [3]), we conclude that given any $r \in[0,1]$ there exists $x$ such that $\int_{x}^{x+r} p(t) d t=F(x+r)-F(x)=0$. In other words, $H\left(G_{0}\right)=[0,1]$. See Corollary 0.4 for a generalization of this result to Euler graphs.

The motivation behind the definition of the chord set also comes from the following combinatorial-analysis theorem.

Theorem 0.2. Suppose $f$ is a Riemann-integrable function on $I=[0,1]$ such that $\int_{I} f(t) d t=0$. Then for any positive integer $k$ there exists a subinterval $J \subseteq I$ of size $1 / k$ such that $\int_{J} f(t) d t=0$. Moreover, if $k$ is replaced by a number that is not a positive integer, the conclusion does not necessarily hold.

In particular, $P(I)=\{1 / k: k \in \mathbb{N}\}$ and $H(I)=\{0,1 / k: k \in \mathbb{N}\}$, where $I$ is viewed as the connected graph with two vertices and one edge. Stronger versions of Theorem 0.2 can be found in [1, 5] in relation with the Horizontal Chord Theorem and the Mountain Climbing Problem. The proof uses a simple continuity argument as follows. Let us define

$$
F(x)=\int_{x}^{x+1 / k} f(t) d t, x \in[0,1-1 / k] .
$$

Then

$$
F(0)+F\left(\frac{1}{k}\right)+\ldots+F\left(1-\frac{1}{k}\right)=\int_{0}^{1} f(t) d t=0 .
$$

Since $F$ is a continuous function, the Intermediate Value Theorem 4 implies that there exists $x \in[0,1-1 / k]$ such that $F(x)=0$, and so $\int_{J} f(t) d t=0$ with $J=[x, x+$ $1 / k]$. To prove the second part of the theorem, let $1 /(k+1)<r<1 / k$. We define a function $f$ on $I$ such that $\int_{I} f(t) d t=0$ but $\int_{J} f(t) d t \neq 0$ for any subinterval of size $r$. Choose intervals $U_{i}, i=0, \ldots, k$, of size $(1 / k-r) / 3$ such that $i / k \in U_{i}$. Define a nonnegative function $g$ on $I$ such that $\int_{U_{i}} g(t)=1 /(k+1)$ and $g(t)=0$ if $t \notin \bigcup_{i} U_{i}$. Let $f(t)=g(t)-1$. Then $\int_{I} f(t) d t=0$. However, if $J \subset I$ is an interval of size $r$ such that $\int_{J} f(t) d t=0$, then we have $\int_{J} g(t) d t=r>1 /(k+1)$. It follows that $J$ intersects with at least two of the $U_{i}$ 's; but then $r=\mu_{G}(J) \geq 1 / k-2(1 / k-r) / 3>r$, which is a contradiction.

In the argument above, a crucial property of $1 / k$ with regard to $I=[0,1]$ is used; namely, there is a partition of $[0,1]$ to subintervals of size $r=1 / k$, i.e. $1 / k \in P(I)$. More generally, we have the following theorem.

Theorem 0.3. For any finite connected graph $G$,

$$
\overline{P(G)} \subseteq H(G)
$$

where $\overline{P(G)}$ is the closure of $P(G)$ in $\mathbb{R}$.

The proof of Theorem 0.3 appears at the end of $\S 2$, where we also show that $X_{r}(G)$ is a path-connected metric space in order to be able to use the Intermediate Value Theorem on $X_{r}(G)$. 
If $G(V, E)$ admits an Euler Circuit, then $P(G)$ is the set of all rational numbers in $(0,|E|]$ and so Theorem 0.3 implies that $H(G)=[0,|E|]$.

Corollary 0.4. If $G(V, E)$ is a graph with an Euler Circuit, then it has every chord, i.e. $\overline{P(G)}=H(G)=[0,|E|]$.

In $\S 3$, we show that the chord set of a connected finite graph with no vertex of degree 1 contains the interval $[0, l(G)]$, where $l(G)$ is the length of the shortest closed path in $G$. In $\S 4$, we generalize Corollary 0.4 to infinite but locally finite graphs.

\section{The SPACE $X_{r}(G)$}

In addition to being a measure space and a topological space, any graph $G$ is equipped with a natural metric. The distance between two points in $G$ is the length of the shortest path connecting them. We extend this metric on $G$ to $X(G)$ by setting:

$$
d_{G}(A, B)=\min \left\{\mu_{G}(C): A \cup B \subseteq C \in X(G)\right\}-\mu_{G}(A \cap B), A, B \in X(G) .
$$

It is straightforward to check that $\left(X(G), d_{G}\right)$ is a metric space. Moreover, $X(G)$ is connected. In fact, any $C \in X(G)$ can be retracted continuously to any $x \in C$, by which we mean:

Lemma 1.1. For any nonempty $C \in X(G)$ and any $x \in C$, there exists a continuous curve $\eta:\left[0, \mu_{G}(C)\right] \rightarrow X(G)$ such that $\eta(0)=C, \eta\left(\mu_{G}(C)\right)=\{x\}$, $\mu_{G}(\eta(t))=\mu_{G}(C)-t$ for all $t$, and $\eta\left(t_{1}\right) \subset \eta\left(t_{2}\right)$ whenever $t_{1}>t_{2}$.

Proof. Suppose $C$ contains a closed path and let $a$ denote the interior of one of the edges in the closed path that does not contain $x$. Then we can retract $C$ to $C \backslash a$ by removing $a$ continuously from $C$ while staying in $X(G)$. By continuing this process, we arrive at a set $C^{\prime} \in X(G)$ which contains $x$ but no closed paths (it is possible that $C=C^{\prime}$ ). Then $C^{\prime}$ has the topology of a tree and it is straightforward to show that $C^{\prime}$ can be retracted to $x$.

The main step in proving Theorem 0.3 is the following theorem.

Theorem 1.2. The metric space $\left(X_{r}(G), d_{G}\right)$ is path-connected for $r \in(0,|E|)$.

Proof. Let $A, B \in X_{r}(G)$ and let $\mathcal{C}$ be the path-connected component of $X_{r}(G)$ that contains $A$. We first show that there exists an element in $\mathcal{C}$ that intersects with $B$. Let

$$
\alpha=\inf _{U \in \mathcal{C}} d_{G}(B, U)
$$

Choose $C \in X_{r}(G)$ such that

$$
d_{G}(B, C)<\alpha+r .
$$

If $B \cap C=\emptyset$, then there exists a continuous curve $\gamma:[0, s] \rightarrow G$ with $\mu_{G}(\gamma([0, t]))=$ $t$, for all $t \in[0, s]$, such that $\gamma(0) \in C$ and $\gamma(s) \in B$, where $s=d_{G}(B, C)-2 r>0$. Let

$$
\theta=\min \{r, s\} .
$$

Let $C_{t}, t \in[0, r]$, be the retraction of $C$ to $\gamma(0) \in C$, obtained in Lemma 1.1. Then

$$
U_{t}=C_{t} \cup \gamma([0, t]), t \in[0, \theta]
$$


is a well-defined continuous curve in $X_{r}(G)$ that connects $C$ to $C_{\theta}$. Now, we have

$$
d_{G}\left(B, C_{\theta}\right) \leq d_{G}(B, C)-\theta=s+2 r-\theta .
$$

If $s>\theta$, then $\theta=r$, and the inequalities (1.2) and (1.3) imply that $d_{G}\left(B, C_{\theta}\right)<\alpha$, which contradicts (1.1). Hence, $s=\theta$ and $d_{G}\left(B, C_{\theta}\right)=2 r$, i.e. $B \cap C_{\theta} \neq \emptyset$.

Next, we fix $x \in B$ such that $\mathcal{C}_{x}=\{C \in \mathcal{C}: x \in B \cap C\} \neq \emptyset$. By the discussion above, we know such an $x$ exists. For each $C \in \mathcal{C}_{x}$, let $U_{C}$ be the connected component of $B \cap C$ that contains $x$ and set

$$
T=C \backslash U_{C}=\bigcup_{i=1}^{k} T_{i}, B \backslash C=\bigcup_{i=1}^{l} B_{i},
$$

where each $B_{i}$ (respectively, $T_{i}$ ) is a connected component of $B \backslash C$ (respectively, $T)$. The numbers $k=k(C)$ and $l=l(C)$ are functions of $C$. Define

$$
\omega=\omega(C)=k(C)+l(C) \geq 0 .
$$

We show that if $\omega(C)>0$, then there exists $C^{\prime} \in \mathcal{C}_{x}$ such that $\omega\left(C^{\prime}\right) \leq \omega(C)-1$. Without loss of generality, we can assume there exists $y \in \overline{B_{1}} \cap U_{C}$. Also there exists $z \in \overline{T_{1}} \cap U_{C}$, since $C$ is connected. Let $\eta:\left[0, \mu_{G}\left(T_{1}\right)\right] \rightarrow G$ be the retraction of $T_{1}$ to $z$ obtained in Lemma 1.1. Similarly, let $\nu:\left[0, \mu_{G}\left(B_{1}\right)\right] \rightarrow G$ be the retraction of $B_{1}$ to $y$. Let

$$
V_{t}=\left(C \backslash\left(T_{1} \backslash \eta(t)\right)\right) \cup \nu\left(\mu_{G}\left(B_{1}\right)-t\right), t \leq \min \left\{\mu_{G}\left(B_{1}\right), \mu_{G}\left(T_{1}\right)\right\} .
$$

Then $V_{t}$ is a continuous curve in $X_{r}(G)$ connecting $C$ to $C^{\prime}=V_{\lambda}$, where $\lambda=$ $\min \left\{\mu_{G}\left(B_{1}\right), \mu_{G}\left(T_{1}\right)\right\}$. If $\lambda=\mu_{G}\left(B_{1}\right)$, then $l\left(C^{\prime}\right) \leq l(C)-1$. On the other hand, if $\lambda=\mu_{G}\left(T_{1}\right)$, then $k\left(C^{\prime}\right) \leq k(C)-1$. In either case:

$$
\omega\left(C^{\prime}\right)=k\left(C^{\prime}\right)+l\left(C^{\prime}\right) \leq \omega(C)-1 .
$$

Now replace $C$ by $C^{\prime}$ and repeat the process above. In a finite number of steps we get $C^{\prime} \in \mathcal{C}$ with $\omega\left(C^{\prime}\right)<1$. But then $k\left(C^{\prime}\right)=0$ and so $B=C^{\prime} \in \mathcal{C}$. This concludes the proof of Theorem 1.2

Now we are ready to present the proof of Theorem 0.3 .

Proof of Theorem 0.3 . Since the chord set is a closed subset of $\mathbb{R}$, it is sufficient to prove $P(G) \subseteq H(G)$. Let $r \in P(G)$ and $f \in L^{1}(G)$ such that $\int_{G} f=0$. We will show that there exists $U \in X_{r}(G)$ such that $\int_{U} f=0$.

Let $I_{f}: X_{r}(G) \rightarrow \mathbb{R}$ be the integration map defined by

$$
I_{f}(A)=\int_{A} f d \mu_{G}
$$

We show that $I_{f}$ is continuous on $X_{r}(G)$ (the topology on $X_{r}(G)$ is induced by the metric $\left.d_{G}\right)$. Suppose $A_{i} \rightarrow A$ in $X_{r}(G)$. Then

$$
\left|I_{g}\left(A_{i}\right)-I_{g}(A)\right|=\left|\int_{A_{i} \oplus A} g d \mu_{G}\right| \rightarrow 0,
$$

and so $I_{f}$ is continuous at every $A \in X_{r}(G)$. Now, since $r \in P(G)$, there exists a collection $\left\{U_{\alpha}\right\}_{1}^{k} \subset X_{r}(G)$ such that almost every point of $G$ appears in exactly $n$ members of the collection. Hence,

$$
\sum_{\alpha=1}^{k} I_{f}\left(U_{\alpha}\right)=\sum_{\alpha=1}^{k} \int_{U_{\alpha}} f d \mu_{G}=n \int_{G} f d \mu_{G}=0 .
$$


If $\exists \alpha, I_{f}\left(U_{\alpha}\right)=0$, then we are done. Otherwise, equation (1.5) implies that $\exists \alpha, \beta$ : $U_{\alpha}<0<U_{\beta}$. By Theorem 1.2 there exists a continuous path $\gamma:[0,1] \rightarrow X_{r}(G)$ such that $\gamma(0)=U_{\alpha}$ and $\gamma(1)=U_{\beta}$. Now the function $I_{f} \circ \gamma$ is continuous and $\gamma(0)<0<\gamma(1)$. It follows from the Intermediate Value Theorem [4] that there exists $t \in(0,1)$ such that $I_{f} \circ \gamma(t)=0$; i.e. for $U=\gamma(t) \in X_{r}(G)$ we have $I_{f}(U)=0$. This completes the proof of Theorem 0.3 .

\section{THE CHORD SET OF FINITE GRAPHS}

In this section, we prove the following theorem.

Theorem 2.1. Suppose $G$ is a finite connected graph (possibly with loops and parallel edges) with no vertex of degree 1 . Let $l(G)$ be the length of the shortest closed path in $G$. Then

$$
[0, l(G)] \subseteq H(G) .
$$

A closed path is called a semi-simple closed path if i) no two consecutive edges along the path are the same and ii) every edge is repeated at most twice along the path. We say a graph $G$ admits a double covering by semi-simple closed paths if there are semi-simple closed paths $C_{i}, i=1, \ldots, k$, that altogether cover every edge of $G$ exactly twice. In other words, every edge in $G$ either belongs to exactly two $C_{i}$ 's but is repeated once in each one of them, or it belongs to only one of the $C_{i}$ 's but is repeated twice. We allow repetitions among the $C_{i}$ 's; i.e. it is possible that $C_{i}$ and $C_{j}$ contain the same edges and $i \neq j$.

It is easily seen that if $G$ contains a vertex of degree one, then $G$ does not admit a double covering by semi-simple closed paths. In order to prove Theorem 2.1, we need to show that the converse is true:

Lemma 2.2. Suppose $G$ is a connected graph such that

$$
\operatorname{deg} v>1, \forall v \in V(G) .
$$

Then $G$ admits a double covering by semi-simple closed paths.

Proof. Let $\mathcal{S}$ denote the set of all subgraphs of $G$ that admit a double covering by semi-simple closed paths. The degree condition (2.1) implies that there exists a simple closed path in $G$. Since every simple closed path is a semi-simple closed path, the set $\mathcal{S}$ is nonempty. Let $K$ be a maximal element of $\mathcal{S}$; i.e. $K$ is not a subgraph of any other element of $\mathcal{S}$. Let $L$ be the graph obtained from $G$ by removing the edges of $K$. Since $K$ is maximal in $\mathcal{S}$, the subgraph $L$ does not contain any closed paths. In particular, there are no loops or parallel edges in $L$. In the sequel, we prove by contradiction that $L$ contains no edges.

Let $\alpha$ be the longest simple path in $L$ and let $u$ and $v$ be the endpoints of $\alpha$. It follows from the degree condition (2.1) and the fact that there are no cycles in $L$ that $u$ and $v$ are distinct vertices of $K$. Since $K \in \mathcal{S}$, there is a collection $M=\left\{C_{i}\right\}_{1}^{k}$ of semi-simple closed paths such that every edge of $G$ appears altogether twice along these paths. In particular, there are semi-simple closed paths $\gamma=C_{i}$ and $\eta=C_{j}$ that contain the vertices $u$ and $v$ respectively. If $i \neq j$, then the path $\beta=\gamma \cdot \alpha \cdot \eta \cdot \overleftarrow{\alpha}$ is a semi-simple path, where $\overleftarrow{\alpha}$ means the reverse of $\alpha$. We can remove $\gamma$ and $\eta$ from the collection $M$ and add $\beta$ to obtain a larger element in $\mathcal{S}$. This contradicts our assumption that $K$ was maximal. If $i=j$, then we partition $\gamma$ into two paths $\gamma_{1}$ and $\gamma_{2}$ such that $\gamma_{1}, \gamma_{2}$ are paths from $u$ to $v$. Now by removing $\gamma$ from the collection $M$ and adding the path $\gamma_{1} \cdot \alpha \cdot \gamma_{2} \cdot \alpha$ we obtain a larger element of $\mathcal{S}$. 
This again contradicts the definition of $K$. It follows that $L$ contains no edges; i.e. $K=G$ and $G$ admits a double covering by semi-simple closed paths.

For a path $P=a_{1} \ldots a_{m}$ and $f \in L^{1}(G)$, we define

$$
\int_{P} f d \mu_{G}=\sum_{i=1}^{m} \int_{a_{i}} f d \mu_{G}
$$

Now we are ready to present the proof of Theorem 2.1.

Proof of Theorem 2.1. Let $r \in[0, l(G)]$ and $f \in L^{1}(G)$ such that $\int_{G} f=0$. By Lemma 2.2 and by the definition of double covering, there exist semi-simple closed paths $C_{1}, \ldots, C_{k}$ such that

$$
\sum_{i=1}^{k} \int_{C_{i}} f d \mu_{G}=2 \int_{G} f d \mu_{G}=0 .
$$

We note that if $C$ is a semi-simple closed path, then there exists $A \in X_{r}(G)$ such that $\int_{A} f=r \int_{C} f$. To see this, suppose the length of $C$ is $n$ and let $F_{n}$ denote the cycle of length $n$. Then there exists a continuous map $\phi: F_{n} \rightarrow C$ that maps the edges of $F_{n}$ to those of $C$. Moreover, we can choose $\phi$ to be measure-preserving when restricted to each edge of $F_{n}$. Since $H\left(F_{n}\right)=[0, n]$, there exists $B \in X_{r}\left(F_{n}\right)$ such that

$$
\int_{B} f \circ \phi(\theta) d \theta=r \int_{F_{n}} f \circ \phi(\theta) d \theta .
$$

On the other hand, we have $r \leq l(G)$, and so $A=\theta(B)$ is connected of size $r$. Moreover, we conclude from (2.3) that $I_{f}(A)=\int_{A} f=r \int_{C} f$.

It follows that for each $i=1, \ldots, k$ there exists $A_{i} \in X_{r}(G)$ such that

$$
I_{f}\left(A_{i}\right)=\int_{A_{i}} f d \mu_{G}=r \int_{C_{i}} f d \mu_{G} .
$$

The equations (2.2) and (2.4) imply that

$$
\sum_{i=1}^{k} I_{f}\left(A_{i}\right)=r \sum_{i=1}^{k} \int_{C_{i}} f d \mu_{G}=0 .
$$

Then the Intermediate Value Theorem implies that there exists a $U \in X_{r}(G)$ such that $I_{f}(U)=0$, and so $r \in H(G)$.

\section{THE CHORD SET OF LOCALLY FINITE GRAPHS}

By a locally finite graph, we mean a graph that may contain infinitely many vertices but the degree of every vertex if finite. If $G(V, E)$ is a connected locally finite graph, then $V$ and $E$ are at most countable. Let $a_{1}, a_{2}, a_{3}, \ldots$ be an enumeration of the edges in $E$. We say $f \in L^{1}(G)$ if

$$
\sum_{i=1}^{\infty} \int_{a_{i}}|f| d \mu_{G}<\infty .
$$

If $f \in L^{1}(G)$, then $\int_{G} f d \mu_{G}$ is defined by

$$
\int_{G} f d \mu_{G}=\sum_{i=1}^{\infty} \int_{a_{i}} f d \mu_{G} .
$$


Clearly this definition is independent of the enumeration of the edges of $G$. In this section, we prove two theorems on locally finite graphs which are analogous to Corollary 0.4 and Theorem 2.1. Note that no infinite graph can have an Euler Circuit. So to generalize Corollary 0.4 to infinite graphs, recall that if the degree of every vertex in a finite connected graph is even, then the graph admits an Euler Circuit. In this section, we first prove the following theorem.

Theorem 3.1. Let $G$ be a connected and locally finite graph with no vertex of odd degree. If $f \in L^{1}(G)$ such that $\int_{G} f=0$, then for any $r \geq 0$, there exists $U \in X_{r}(G)$ such that $\int_{U} f=0$.

Proof. The proof is by contradiction. Suppose that for every $U \in X_{r}(G), I_{f}(U) \neq$ 0 . Then the function $I_{f}(U)=\int_{U} f$ has a fixed sign, say positive, on $X_{r}(G)$.

Let $v \in G$ be a fixed vertex. Define $G_{k}$ to be the maximal subgraph consisting of the vertices of distance at most $k$ from $v$. Then $G_{k}$ is a connected finite graph with an even number of odd-degree vertices $v_{1}, \ldots, v_{m}$ (it is possible that $m=0$ ). Since every vertex in $G$ has even degree, each $v_{i}$ must be exactly at distance $k$ from $v$. We construct a new graph $G_{k}^{\prime}$ by connecting $v_{2 j-1}$ to $v_{2 j}$ via an edge $a_{j}$ for $j=1, \ldots, m / 2$. Every vertex in $G_{k}^{\prime}$ has even degree and, moreover, $G_{k}^{\prime}$ is connected. It follows that $G_{k}^{\prime}$ is an Euler graph. Using the Euler Circuit of $G_{k}^{\prime}$, one can partition $G_{k}^{\prime}$ to connected closed subsets $J_{1}, J_{2}, \ldots, J_{l}$ such that each intersection $J_{i} \cap J_{j}, 1 \leq i<j \leq l$, consists of at most a finite number of points and

$$
\forall j<l:\left|J_{j}\right|=r, 0<\left|J_{l}\right| \leq r .
$$

For $k$ large enough, without loss of generality, we can assume $v \in J_{1}$ and $v_{1} \in J_{l}$. Define

$$
S=\left\{1 \leq j \leq l-1 \mid J_{j} \subseteq G_{k}\right\}, T=\left\{j \notin S \mid J_{j} \cap G_{k} \neq \emptyset\right\} .
$$

Also let

$$
E_{k}=\bigcup_{j \in T} J_{j}
$$

Next, let $X_{r}(G, v)$ denote the set of connected subsets of $G$ of size $r$ containing $v$. Then $X_{r}(G, v)$ is a compact set, and so $\left.I_{f}\right|_{\Gamma_{r}}$ has a positive lower bound that we denote by $\epsilon$. It follows from the positivity of $I_{f}$ on $X_{r}(G)$ that

$$
\sum_{j \in S} \int_{J_{j}} f d \mu_{G}=\sum_{j \in S} I_{f}\left(J_{j}\right) \geq I_{f}\left(J_{1}\right) \geq \epsilon .
$$

Since $J_{j} \nsubseteq G_{k}$ for all $j \in T$, we have $\operatorname{dist}_{G}(v, x) \geq k-r$ for all $x \in E_{k}$. In other words,

$$
\operatorname{dist}_{G}\left(v, E_{k}\right) \geq k-r .
$$

It follows from the integrability of $f$ and (3.3) that $\int_{E_{k} \cap G_{k}} f d \mu_{G}$ converges to zero as $k \rightarrow \infty$, which together with (3.2) implies that

$$
\int_{G_{k}} f d \mu_{G}=\sum_{j \in S} \int_{J_{j}} f d \mu_{G}+\int_{E_{k} \cap G_{k}} f d \mu_{G} \geq \frac{\epsilon}{2},
$$

for $k$ large enough. This contradicts the assumption that $\int_{G} f=0$, and the theorem follows.

We now state and prove the analogue of Theorem 2.1 for connected locally finite graphs. 
Theorem 3.2. Suppose $G(V, E)$ is a connected locally finite graph such that

$$
\operatorname{deg} v>1, \forall v \in V \text {. }
$$

Then $[0,1] \subset H(G)$.

Proof. As in the proof of Theorem 3.1, we assume $I_{f}$ is positive on $X_{r}(G)$, where $r \in[0,1]$, and we derive a contradiction. Recall that $X_{r}(G, v)$ is the set of connected closed subsets of $G$ of size $r$ that contain $v$. Also $\epsilon$ is the lower bound of $I_{f}$ on $X_{r}(G, v)$. We need the following lemma:

Lemma 3.3. Suppose $I_{f}$ is positive on $X_{r}(G)$ and bounded below by $\epsilon$ on $X_{r}(G, v)$, where $r \in[0,1]$. If $C$ is a semi-simple closed path in $G$, then $\int_{C} f d \mu_{G}>0$. If moreover $C$ contains $v$, then $\int_{C} f d \mu_{G} \geq \epsilon$.

We postpone the proof of this lemma and continue with the proof of Theorem 3.2 . Define $G_{k}$ to be the maximal subgraph consisting of the vertices of distance at most $k$ from $v$, where $k \in \mathbb{N}$. Then $G_{k}$ is a connected finite graph with a finite number of vertices of degree 1 . Let $v_{1}, \ldots, v_{m}$ be the set of vertices of $G_{k}$ of degree 1 (it is possible that $m=0$ ). Since the degree of every vertex in $G$ is greater than 1, each $v_{i}$ must be exactly at distance $k$ from $v$. We construct a new graph $G_{k}^{\prime}$ by attaching a loop at each vertex $v_{i}$ and extend $f$ by zero to $G_{k}^{\prime}$. Since $G_{k}^{\prime}$ is connected and the degree of every vertex in $G_{k}^{\prime}$ is greater than 1 , we conclude from Lemma 2.2 that $G_{k}^{\prime}$ admits a double covering by a collection $\mathcal{U}_{k}$ of semi-simple closed paths. For $i \leq m$, let $C_{i} \in \mathcal{U}_{k}$ be the unique path in the collection $\mathcal{U}_{k}$ that contains the attached loop at $v_{i}$.

Similar to the proof of Theorem 3.1, we cover each $C_{i}$ with a collection of connected closed subsets of $G_{k}^{\prime}$ satisfying conditions (3.1). Having done this covering for each $i \leq m$, we arrive at a collection $U_{1}, \ldots, U_{\alpha}, V_{1}, \ldots, V_{\beta}$ of closed and connected subsets of $G_{k}^{\prime}$ with the following properties:

i) $U_{i} \in X_{r}(G)$ for all $i \leq \alpha$,

ii) $\mu_{G}\left(V_{i}\right) \leq r$ and $\operatorname{dist}_{G}\left(v, V_{i}\right) \geq k-1$ for all $i \leq \beta$.

Since $\operatorname{dist}_{G}\left(v, V_{i}\right) \geq k-1$, we conclude from the integrability of $f$ on $G$ that

$$
\lim _{k \rightarrow \infty} \sum_{i=1}^{\beta} \int_{V_{i}} f d \mu_{G}=0 .
$$

Let $\mathcal{V}_{k}=\mathcal{U}_{k} \backslash\left\{C_{i}: i \leq m\right\}$. Either $v \in U_{i}$ for some $i \leq \alpha$, or $v \in C$ for some $C \in \mathcal{V}_{k}$, and so

$$
\sum_{i=1}^{\alpha} I_{f}\left(U_{i}\right)+\sum_{C \in \mathcal{V}_{k}} I_{f}(C) \geq \epsilon
$$

Now consider the equality below:

$2 \int_{G_{k}} f d \mu_{G}=\sum_{i=1}^{m} \int_{C_{i}} f d \mu_{G}+\sum_{C \in \mathcal{V}_{k}} I_{f}(C)=\sum_{i=1}^{\alpha} I_{f}\left(U_{i}\right)+\sum_{i=1}^{\beta} \int_{V_{i}} f d \mu_{G}+\sum_{C \in \mathcal{V}_{k}} I_{f}(C)$.

Taking the limit as $k \rightarrow \infty$, the left-hand side converges to 0 while the right-hand side is bounded from below by $\epsilon$ by (3.5) and (3.6). This is a contradiction, and the theorem follows.

It is left to prove Lemma 3.3 . 
Proof of Lemma 3.3. We first prove that if $g: \mathbb{R} \rightarrow \mathbb{R}$ is a locally integrable periodic function with period $L$ and $F: \mathbb{R} \rightarrow \mathbb{R}$ is defined by

$$
K(x)=\int_{x}^{x+r} g(t) d t
$$

then

$$
\int_{0}^{L} K(x) d x=r \int_{0}^{L} g(t) d t .
$$

Define the auxiliary function $h(x, y)=g(y)$ on $\mathbb{R}^{2}$. Let $D$ be the region

$$
D=\left\{(x, y) \in \mathbb{R}^{2} \mid x \in[0, L], y \in[x, x+r]\right\} .
$$

We have

$$
\int_{D} h(x, y) d y d x=\int_{0}^{L} \int_{x}^{x+r} g(y) d y d x=\int_{0}^{L} K(x) d x .
$$

On the other hand, by Fubini's Theorem:

$$
\begin{aligned}
\int_{D} h(x, y) d x d y & =\int_{0}^{r} \int_{0}^{y} g(y) d x d y+\int_{r}^{L} \int_{y-r}^{y} g(y) d x d y+\int_{L}^{L+r} \int_{y-r}^{L} g(y) d x d y \\
& =\int_{0}^{r} y g(y) d y+r \int_{r}^{L} g(y) d y+\int_{L}^{L+r}(L+r-y) g(y) d y \\
& =\int_{0}^{r} y g(y) d y+r \int_{r}^{L} g(y) d y+\int_{0}^{r}(r-y) g(y) d y \\
& =r \int_{0}^{L} g(y) d y
\end{aligned}
$$

where we used the change of variables $y \rightarrow y-L$ and that $g(y)=g(y-L)$ by periodicity of $g$ to simplify $\int_{L}^{L+r}(L+r-y) g(y) d y$. This completes the proof of equation (3.8).

Now let $C$ be a semi-simple closed path in $G$ of length $L$ and let $\phi: F_{L} \rightarrow C$ be as in the proof of Theorem 2.1] i.e. $F_{L}$ is the cycle of length $L$ and $\phi$ maps the edges of $F_{L}$ onto those of $C$ continuously such that the restriction of $\phi$ on each edge of $F_{L}$ is measure-preserving. We can think of $F_{L}$ as the interval $[0, L]$ on $\mathbb{R}$ by identifying 0 and $L$. Let

$$
g(t)=f \circ \phi(t), t \in[0, L],
$$

and extend $g$ to $\mathbb{R}$ such that $g(t+L)=g(t)$ for all $t \in \mathbb{R}$. Let $K$ be defined as in (3.7). Note that $K(x)=I_{f}(\phi([x, x+r]))>0$ for all $x$, since $I_{f}$ is assumed to be positive on $X_{r}(G)$. Then equation (3.8) implies that

$$
\int_{C} f d \mu_{G}=\int_{0}^{L} g(t) d t=\frac{1}{r} \int_{0}^{L} K(x) d x>0 .
$$

Finally, we consider the case where $v \in C$. Without loss of generality, assume that $\phi(1)=v$ (recall that we have identified $F_{L}$ with $\left.[0, L]\right)$. Then $K(x) \geq \epsilon$ for all $x \in[1-r, 1]$. Equation (3.8) implies that, in this case,

$$
\int_{C} f d \mu_{G}=\int_{0}^{L} g(t) d t=\frac{1}{r} \int_{0}^{L} K(x) d x \geq \frac{1}{r} \int_{1-r}^{1} K(x) d x \geq \frac{1}{r} \cdot r \cdot \epsilon \geq \epsilon .
$$

This completes the proof of Lemma 3.3 . 


\section{REFERENCES}

1. Contests in Higher Mathematics, 1949-1961, Akadémiai Kiadó, Budapest, 1968. MR0239895 $(39: 1252)$

2. J.P. Huneke, Mountain Climbing, Trans. Amer. Math. Soc. 139 (1969) 383-391. MR0239013 $(39: 372)$

3. J.C. Oxtoby, Horizontal Chord Theorem, Amer. Math. Monthly 79 (1972) 468-475. MR 0299735 (45:8783)

4. K.A. Ross, Elementary Analysis: The Theory of Calculus, Springer-Verlag, 1980. MR560320 (81a:26001)

5. V. Totik, A Tale of Two Integrals, Amer. Math. Monthly 106 (1999) 227-240. MR:1682343 (2000d:26002)

Department of Mathematics, University of Oregon, Eugene, Oregon 97403

E-mail address: javaheri@uoregon.edu

Current address: Department of Mathematics, Trinity College, 300 Summit Street, Hartford, Connecticut 06106

E-mail address: Mohammad.Javaheri@trincoll.edu 